\title{
The chemical structure of the hot pulsating DB white dwarf KIC 08626021 from asteroseismology
}

\author{
S. Charpinet ${ }^{1}$, P. Brassard ${ }^{2}$, N. Giammichele ${ }^{1}$, \\ and Gilles Fontaine ${ }^{2}$
}

${ }^{1}$ Institut de Recherche en Astrophysique et Planétologie, CNRS, Université de Toulouse, CNES, 14 avenue Edouard Belin, F-31400 Toulouse, France

email: stephane. charpinet@irap. omp.eu

${ }^{2}$ Département de Physique, Université de Montréal, Québec H3C 3J7, Canada

\begin{abstract}
Giammichele et al. (2018) proposed a full determination, largely independent of evolution calculations, of the chemical composition and stratification inside the hot pulsating DB white dwarf KIC 08626021. However, Timmes et al. (2018) pointed out that neglecting the effects of neutrino cooling, such as in the static models used in Giammichele et al. study, could impact significantly the derived seismic solution and compromise conclusions drawn upon it. Here we present a reanalysis of KIC 08626021, using improved static models which now incorporate more realistic luminosity profiles that reflect the still significant energy losses induced by neutrino emission mechanisms in hot DB white dwarfs. We show that this effect has only a limited impact on the derived seismic model properties and, more importantly, that all the conclusions brought by Giammichele et al. (2018) remain entirely valid.
\end{abstract}

Keywords. white dwarfs, stars: interiors, stars: oscillations

\section{Context}

Giammichele et al. (2018) established from asteroseismology, using a new flexible parameterization of the chemical stratification in white dwarf cores (Giammichele et al. 2017a,b), that the pulsating DB white dwarf KIC 08626021 has a central homogeneous core $\sim 15 \%$ richer in oxygen (in mass) and $\sim 40 \%$ more massive than predicted by standard evolutionary models. This finding provides strong and challenging constraints to the modeling of stellar cores during the helium-burning phase that precedes (e.g., Fields et al. 2016; De Gerónimo et al. 2017, 2018). However, this result has been questioned by Timmes et al. (2018), who pointed out that the impact of neutrino cooling on the thermal structure of the star - an effect neglected in the static equilibrium models employed by Giammichele et al. (2018) - is still significant in a hot DB white dwarf like KIC 08626021. They found that low-order $g$-mode frequencies could differ by as much as $\sim 70 \mu \mathrm{Hz}(\sim 30 \mu \mathrm{Hz}$ on average) between hot DB white dwarf models neglecting and including neutrino losses. Such differences could induce significant shifts in the derived parameters of KIC 08626021, possibly changing the conclusions drawn upon its seismically determined structure. We have therefore undertaken a reanalysis of this star, now accounting properly for the effects of neutrino emission. Details on this new analysis are published in Charpinet et al. (2019) and briefly described below. 


\section{New static models with neutrinos}

The models used in Giammichele et al. (2018) are static structures constructed on the assumption that the luminosity profile is proportional to the mass distribution $\left(L_{r} \propto\right.$ $M_{r}$; hereafter referred to as the " 0 th-order" approximation). This assumption is well verified in white dwarfs that have cooled sufficiently, such as DA pulsators found in the $T_{\text {eff }}=13,000-11,000 \mathrm{~K}$ range. However, for hot white dwarfs, such as the heliumatmosphere DB pulsators found in the $T_{\text {eff }}=32,000-22,000 \mathrm{~K}$ range, cooling from neutrino emission is still important, with a significant impact on the thermal structure and pulsation frequencies, as illustrated by Timmes et al. (2018). The above assumption on the luminosity profile is therefore no longer appropriate in that case.

To address this problem, we introduce improved static models that are constructed from the relation $L_{r}=M_{r} \cdot\left(L_{*} / M_{*}-k_{\nu}\right)$, where $k_{\nu}$ accounts for all effects (mainly neutrino cooling, but also residual contraction) that would cause the luminosity profile to differ from the $L_{r} \propto M_{r}$ relation. The quantity $k_{\nu}$ is interpolated from a grid of luminosity profiles obtained from "generic" white-dwarf cooling sequences (evolutionary models) covering a wide range of values for $T_{\text {eff }}$, mass, He-envelope thickness, and core composition ( $\mathrm{C} / \mathrm{O}$ relative mass fraction). These models are generic in the sense that their structure is a simple $\mathrm{C} / \mathrm{O}$ core of homogeneous composition surrounded by a pure helium envelope, providing the main, " 1 st - order" correction to the luminosity profile. A " $2^{\text {nd }}$ order" correction accounting for a particular chemical stratification, e.g., as inferred from a seismic model solution, can be obtained if necessary, by calibrating the interpolation of $k_{\nu}$ relative to a specific cooling sequence. This approach produces improved static white-dwarf models that satisfactorily account for the impact of neutrino cooling (and other less important effects) on the thermal structure (see Charpinet et al. 2019).

When comparing adiabatic frequencies for relevant $g$-modes computed from two identical models representative of KIC 08626021, one including the effects of neutrino emission as described above, and the other neglecting them, we find frequency differences that are indeed comparable to the variations reported by Timmes et al. (2018).

\section{Updated seismic solution for KIC 08626021}

KIC 08626021 was reanalysed with our updated static models, following an iterative approach as described in Charpinet et al. (2019), in order to achieve a " $2^{\text {nd }}$-order" solution. Compared to Giammichele et al. (2018), we find that using the new improved models has no negative impact on the achieved quality of the optimal fit. They lead to the identification of a very well defined seismic solution that also reproduces the frequencies of KIC 08626021 to the precision of the observations $(\sim 0.6 \mathrm{nHz})$ and that does not induce changes in the mode identification. The new solution is however shifted relative to the former model uncovered by Giammichele et al. (2018). The most relevant parameters of this updated solution are given in Table 1 and Fig. 1 shows the new chemical stratification uncovered compared to the former solution of Giammichele et al. (2018).

We first find that the new improved solution for KIC 08626021 remains close to the former model, as far as global stellar parameters $\left(T_{\text {eff }}, \log g, M_{*}\right.$, and $\left.R_{*}\right)$ are concerned. Shifts in these parameters remain close to the $1 \sigma$-uncertainties quoted by Giammichele et al. (2018). Therefore, systematic errors introduced by neglecting neutrino cooling do not propagate evenly among the optimized model parameters. Larger variations appear when comparing the internal stratifications of the new optimal model with the former one, but also impacting unevenly the characteristics of the profiles. The chemical composition in the intermediate $\mathrm{C} / \mathrm{O}$ and $\mathrm{C} / \mathrm{He}$ layers are the most affected. We find that the helium 
Table 1. Updated properties of KIC 08626021 when accounting for neutrino cooling in the seismic models (this work; Charpinet et al. 2019) compared to the former solution from Giammichele et al. (2018).

\begin{tabular}{lcc}
\hline \hline Quantity & Giammichele et al. (2018) & $\begin{array}{c}\text { Charpinet et al. }(2019) \\
\text { (This work) }\end{array}$ \\
\hline $\log g\left(\mathrm{~cm} \mathrm{~s}^{-2}\right)$ & $7.92 \pm 0.01$ & $7.905 \pm 0.014$ \\
$T_{\text {eff }}(\mathrm{K})$ & $29,968 \pm 198$ & $30,114 \pm 210$ \\
$X(\mathrm{He})_{\text {env }}$ & $0.18 \pm 0.04$ & $0.38 \pm 0.07$ \\
$\log \left(q_{1}\right)$ & $-7.63 \pm 0.20$ & $-7.83 \pm 0.36$ \\
$\log \left(q_{2}\right)$ & $-3.23 \pm 0.10$ & $-3.48 \pm 0.07$ \\
$X(\mathrm{O})_{\text {centre }}$ & $0.86 \pm 0.04$ & $0.84 \pm 0.04$ \\
$M(\mathrm{He}) / M_{*}(\%)$ & $0.0113 \pm 0.006$ & $0.0133 \pm 0.0063$ \\
$M(\mathrm{C}) / M_{*}(\%)$ & $21.96 \pm 4.2$ & $20.76 \pm 1.1$ \\
$M(\mathrm{O}) / M_{*}(\%)$ & $78.03 \pm 4.2$ & $79.23 \pm 1.1$ \\
$M_{*} / M_{\odot}$ & $0.570 \pm 0.005$ & $0.562 \pm 0.006$ \\
$R_{*} / R_{\odot}$ & $0.0138 \pm 0.0001$ & $0.0139 \pm 0.0001$ \\
& & \\
\hline
\end{tabular}

mass fraction in the mixed C/He layer, $X(\mathrm{He})_{\text {env }}$, is now $0.38 \pm 0.07$ instead of $0.18 \pm 0.04$, while the amount of oxygen (in mass fraction) in the intermediate $\mathrm{C} / \mathrm{O}$ layer reaches $\sim 0.6$ instead of $\sim 0.4$. In contrast, the other important characteristics of the chemical profiles remain nearly unchanged. In particular, all chemical transitions are found to be located nearly at the same fractional mass depths, except for a slight outward shift of the coreto-envelope transition $\left(\log q_{2}\right)$. The inner core, for its part, remain nearly the same with the central oxygen mass fraction, $X(\mathrm{O})_{\text {centre, }}$ estimated at $0.84 \pm 0.04$ (i.e., very close to the former, $0.86 \pm 0.04$, value) and the homogeneous central part extending up to a fractional mass depth of $\log q \sim 0.71$ (i.e., encompassing a mass of $\sim 0.45 M_{\odot}$ ), which

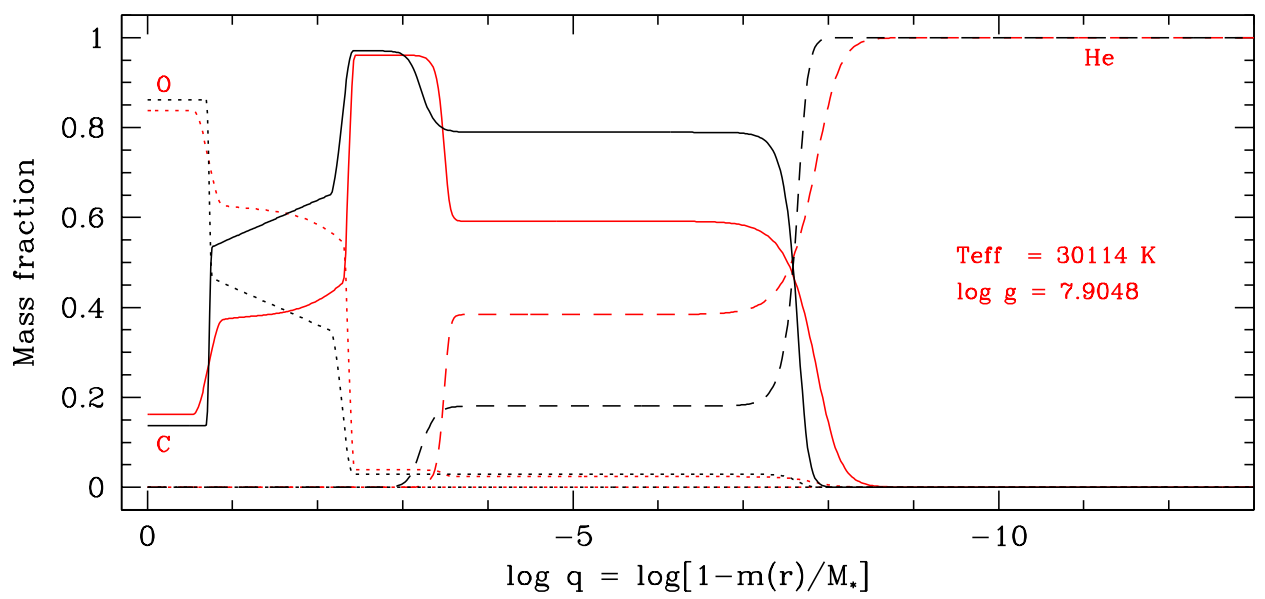

Figure 1. Stratification in helium, carbon, and oxygen derived from the new optimal seismic solution obtained with models incorporating neutrino cooling (red curves) and compared with the former solution from Giammichele et al. (2018; black curves). This plot is taken from Figure 2 of Charpinet et al. (2019). 
is identical to the value of $\log q \sim 0.7\left(\sim 0.45 M_{\odot}\right)$ found from the former solution. Consequently, all the conclusions regarding the inner core size and its $\mathrm{C} / \mathrm{O}$ composition ratio expressed in Giammichele et al. (2018) are unchanged.

\section{Conclusion}

Despite some changes in the determined internal structure, we find that including the effects of neutrino cooling in our static DB white dwarf models has little impact on the main characteristics of the optimal seismic solution for KIC 08626021. The only significantly affected quantities are the $\mathrm{C} / \mathrm{O}$ and $\mathrm{C} / \mathrm{He}$ mass fractions in the intermediate layers of the star. Other quantities, such as the location of helium transitions in the envelope, or the central oxygen mass fraction and extent of the homogeneous central part of the core are either unchanged or only slightly displaced. Therefore, despite significant effects on calculated frequencies induced by omitting neutrinos and their impact on the thermal structure of the star, we find that all conclusions and implications drawn from the analysis of Giammichele et al. (2018) remain completely valid.

\section{Acknowledgements}

We dedicate this work to our inspiring friend and colleague Gilles Fontaine, gone too early. Stéphane Charpinet and Noemi Giammichele acknowledge financial support from the Centre National d'Études Spatiales (CNES, France) and from the Agence Nationale de la Recherche (ANR, France) under grant ANR-17-CE31-0018, funding the INSIDE project. This work was granted access to the high-performance computing resources of the CALMIP computing centre under allocation number 2019-p0205. P.B. and G.F. acknowledge the contribution of the Canada Research Chair Program.

\section{References}

Charpinet, S., Brassard, P., Giammichele, N., \& Fontaine, G. 2019, A\&\&A (Letters), 628, L2

De Gerónimo, F. C., Althaus, L. G., Córsico, A. H., Romero, A. D., \& Kepler, S. O. 2017, A\& A, 599, A21

De Gerónimo, F. C., Althaus, L. G., Córsico, A. H., Romero, A. D., \& Kepler, S. O. 2018, A\&A, 613, A46

Fields, C. E., Farmer, R., Petermann, I., Iliadis, C., \& Timmes, F. X. 2016, ApJ, 823, 46

Giammichele, N., Charpinet, S., Fontaine, G., \& Brassard, P. 2017a, ApJ, 834, 136

Giammichele, N., Charpinet, S., Brassard, P., \& Fontaine, G. 2017b, A\&GA, 598, A109

Giammichele, N., Charpinet, S., Fontaine, G., et al. 2018, Nature, 554, 73

Timmes, F. X., Townsend, R. H. D., Bauer, E. B., et al. 2018, ApJ (Letters), 867, L30 